\title{
Multivariate Regularized Newton and Levenberg-Marquardt methods: a comparison on synthetic data of tumor hypoxia in a kinetic framework
}

\author{
Sara Garbarino $^{1 *}$, Giacomo Caviglia ${ }^{2}$ \\ ${ }^{1}$ Centre for Medical Image Computing, University College London, London, UK \\ ${ }^{2}$ Dipartimento di Matematica, Università di Genova, Italy \\ *Email address for correspondence: s.garbarino@ucl.ac.uk \\ Communicated by Michele Piana and Luigi Preziosi \\ Received on 11 20, 2016. Accepted on 07 24, 2017.
}

\begin{abstract}
In this paper we propose a new algorithm to optimize the parameters of a compartmental problem describing tumor hypoxia. The method is based on a multivariate Newton approach, with Tikhonov regularization, and can be easily applied to data with diverse statistical distributions. Here we simulate $\left[{ }^{18} F\right]$-fluoromisonidazole Positron Emission Tomography dynamic data of hypoxia of a neck tumor and describe the tracer flow inside tumor with a two-compartments compartmental model. We perform optimization on the parameters of the model via the proposed Multivariate Regularized Newton method and validate it against results obtained with a standard Levenberg-Marquardt approach. The proposed algorithm returns parameters that are closer to the ground truth while preserving the statistical distribution of the data.
\end{abstract}

Keywords: Compartmental analysis, Newton methods, tumor hypoxia, Fmiso-PET

AMS subject classification: 65L09, 92C45

\section{Introduction}

$\left[{ }^{18} \mathrm{~F}\right]$-fluoromisonidazole (Fmiso) is largely used for Positron Emission Tomography (PET), mainly in the case of oncological applications. In fact, Fmiso-PET is able to measure hypoxia in tumours [1,2], that has been associated with poor treatment outcome and survival [3,4]. The first kinetic compartmental model describing the transport and metabolism of Fmiso is due to Casciari et al [5], whose model consists of four compartments with a high number of free parameters. Hence, a certain number of parameters have to be fixed a priori to increase the robustness of the model. A more recent work from Thorwarth et al [6] presented a new compartmental system for Fmiso-PET data, in which the kinetic model assumes physical, purely diffusive transport of the tracer molecule to the hypoxic cell, where it is trapped according to the local oxygen tension. The model consists of two compartments and four kinetic parameters; it is identifiable (according to [7]) and the fitting is performed via a Levenberg-Marquardt (LM) approach [8]. In this work we present a new algorithm to reduce such compartmental model, based on a Multivariate Regularized Newton (MRN) method [9]. This method utilizes inverse problems techniques to determine a number of tracer coefficients in a numerically stable fashion. From a mathematical perspective, a fundamental advantage of our scheme with respect to the LM approach, is that the MRN methods do not need to select a priori a topology in the data space, as in the case of least-squares approaches.

The plan of the paper is as follows. Section 2 recalls the two-compartment model for Fmiso-PET metabolism and describes the numerical method for model reduction, while Section 3 shows the numerical and experimental validation of the approach. Our conclusions are offered in Section 4. 


\section{Materials and methods}

\subsection{The compartmental model}

Following the model originated in [6], the Fmiso-PET compartmental problem is described as a system of two compartments which, in contrast to classic compartmental models, are spatially separated or overlap only partially, and occupy different volumes. In addition, some of the kinetic constants of this model are not parameters of a chemical reaction, but reflect purely diffusive transport.

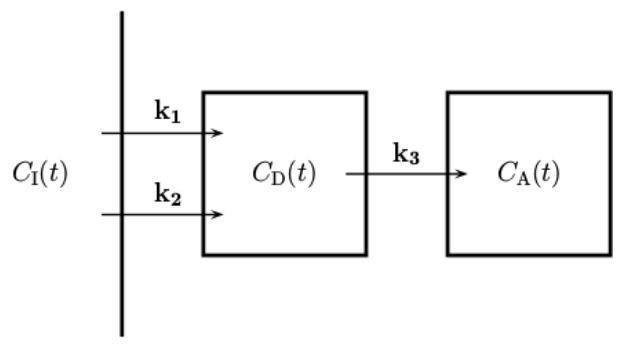

Figure 1. Compartmental model consisting of a diffusive $\left(C_{\mathrm{D}}\right)$ and an accumulative $\left(C_{\mathrm{A}}\right)$ compartment. $C_{\mathrm{I}}$ represent the tracer concentration in the blood and is named Input Function (IF).

The state variables of the two-compartmental model described in Figure 1 are the tracer concentration in $C_{\mathrm{D}}$ and in $C_{\mathrm{A}}$; the kinetic process in the system is initialized by the Input Function (IF) $C_{\mathrm{I}}$, representing the tracer concentration in blood. The three constant coefficients between compartments in contact are denoted as $k_{1}, k_{2}, k_{3}$ and we assume that $k_{i} \geq 0$ for all $i=1,2,3$. Conservation of tracer exchanged between compartments leads to the following system of linear ordinary differential equations with constant coefficients:

$$
\left\{\begin{array}{l}
\dot{C}_{\mathrm{D}}(t)=-\left(k_{2}+k_{3}\right) C_{\mathrm{D}}(t)+k_{1} C_{\mathrm{I}}(t) \\
\dot{C}_{\mathrm{A}}(t)=k_{3} C_{\mathrm{D}}(t)
\end{array}\right.
$$

with initial conditions $C_{\mathrm{A}}(0)=C_{\mathrm{D}}(0)=0$. Equations (1) can be written in the compact form

$$
\dot{\mathbf{C}}=\mathbf{M C}+k_{1} \mathbf{W},
$$

with initial condition $\mathbf{C}(0)=0$ and where

$$
\begin{gathered}
\mathbf{C}:=\left[\begin{array}{l}
C_{\mathrm{D}} \\
C_{\mathrm{A}}
\end{array}\right], \\
\mathbf{M}=\left[\begin{array}{cc}
-\left(k_{2}+k_{3}\right) & 0 \\
k_{3} & 0
\end{array}\right],
\end{gathered}
$$

and

$$
\mathbf{W}:=\left[\begin{array}{c}
C_{\mathrm{I}} \\
0
\end{array}\right]
$$

The analytical solution of Eq. (2) is

$$
\mathbf{C}(t)=k_{1} \int_{0}^{t} C_{\mathrm{I}}(u) e^{(t-u) \mathbf{M}} \mathbf{e}_{\mathbf{1}} d u
$$

where $\mathbf{e}_{\mathbf{1}}=\left[\begin{array}{l}1 \\ 0\end{array}\right]$. Specifically, we have

$$
C_{\mathrm{D}}(t)=k_{1} \int_{0}^{t} C_{\mathrm{I}}(u) e^{-\left(k_{2}+k_{3}\right)(t-u)} d u
$$


and

$$
C_{\mathrm{A}}(t)=\frac{k_{1} k_{3}}{k_{1}+k_{3}} \int_{0}^{t} C_{\mathrm{I}}(u)\left(1-e^{-\left(k_{2}+k_{3}\right)(t-u)}\right) d u
$$

On the basis of these equations compartmental analysis aims at determining the tracer coefficients $k_{1}, k_{2}, k_{3}$ by utilizing measurements of the tracer concentration provided by nuclear imaging and applying an optimization scheme. In nuclear imaging experiments, the reconstructed images can provide information on the tracer concentration in the tissue under examination (typically an organ of interest) as well as in the input arterial blood (that provides the Input Function $C_{\mathrm{I}}$ ) as measured in the left ventricle. Specifically, an acquisition sequence is set up providing count data sets collected at subsequent time intervals. For each data set, an image reconstruction algorithm is applied (Ordered Subset Expectation Maximization, see [10], Regions of Interest (ROIs) are drawn within the left ventricle and the organ of interest, and the corresponding tracer concentrations are computed. In this specific case, the total measured PET signal $S(t)$ is given by a linear combination of the concentrations in the diffusive and accumulative compartments, plus the tracer carried by the blood contained in the tumor's vascular system. Therefore $S(t)$ is a weighted sum of concentrations:

$$
\begin{aligned}
S(t)= & w_{0} C_{\mathrm{I}}(t)+w_{\mathrm{D}} C_{\mathrm{D}}(t)+w_{\mathrm{A}} C_{\mathrm{A}}(t) \\
= & w_{0} C_{\mathrm{I}}(t)+w_{\mathrm{D}} k_{1} \int_{0}^{t} C_{\mathrm{I}}(u) e^{-\left(k_{2}+k_{3}\right)(t-u)} d u \\
& +w_{\mathrm{A}} \frac{k_{1} k_{3}}{k_{1}+k_{3}} \int_{0}^{t} C_{\mathrm{I}}(u)\left(1-e^{-\left(k_{2}+k_{3}\right)(t-u)}\right) d u .
\end{aligned}
$$

Here $w_{\mathrm{D}}$ and $w_{\mathrm{A}}$ are the weights of the diffusive and the accumulative compartment, respectively, and correspond to the volume fraction occupied by these compartments. The weight $w_{0}$ represents the blood fraction with which the tumor's vascular system is supplied. By defining

$$
\begin{aligned}
& \tilde{w}_{\mathrm{D}}=k_{1} w_{\mathrm{D}}, \\
& \tilde{w}_{\mathrm{A}}=\frac{k_{1} k_{3}}{k_{1}+k_{3}} w_{\mathrm{A}},
\end{aligned}
$$

and

$$
\tilde{k}_{3}=k_{2}+k_{3}
$$

equation (9) can be rewritten as

$$
\begin{aligned}
S(t) & =w_{0} C_{\mathrm{I}}(t)+\tilde{w}_{\mathrm{D}} \int_{0}^{t} C_{\mathrm{I}}(u) e^{-\tilde{k}_{3}(t-u)} d u+\tilde{w}_{\mathrm{A}} \int_{0}^{t} C_{\mathrm{I}}(u)\left(1-e^{-\tilde{k}_{3}(t-u)}\right) d u \\
& =w_{0} C_{\mathrm{I}}(t)+\tilde{w}_{\mathrm{A}} \int_{0}^{t} C_{\mathrm{I}}(u) d u+\left(\tilde{w}_{\mathrm{D}}-\tilde{w}_{\mathrm{A}}\right) \int_{0}^{t} C_{\mathrm{I}}(u) e^{-\tilde{k}_{3}(t-u)} d u .
\end{aligned}
$$

The change from $\left(k_{1}, k_{2}, k_{3}, w_{0}, w_{D}, w_{A}\right)$ to the new set of parameters can be performed without loss of generality, since the rate constant $k_{1}$ reflects just a multiplicative factor for $w_{\mathrm{D}}$ and $w_{\mathrm{A}}$ (thus can be set constant) and variation of this parameter are absorbed into $\tilde{w}_{\mathrm{D}}$ and $\tilde{w}_{\mathrm{A}}$. Similarly, $k_{2}$ can be absorbed by $\tilde{k}_{3}$. The resulting kinetic model depends on four parameters: the weights $w_{0}, \tilde{w}_{\mathrm{D}}, \tilde{w}_{\mathrm{A}}$, and the rate constant $\tilde{k}_{3}$. 


\subsection{Multivariate Regularized Newton method}

Consider equation (11) where $S$ and $C_{I}$ are identified by PET image data. The numerical reduction of the two-compartment model under investigation requires the numerical solution of equation (11) by means of an optimization-regularization method. In order to formally describe this inverse problem we introduce the non-linear operator $\mathcal{S}_{t}$

$$
\begin{aligned}
\mathcal{S}_{t}: \mathbb{R}_{+}^{4} & \rightarrow C^{1}\left(\mathbb{R}_{+}, \mathbb{R}\right) \\
\mathbf{k} & \mapsto S_{t}(\mathbf{k})
\end{aligned}
$$

such that

$$
\begin{aligned}
\mathcal{S}_{t}(\mathbf{k})= & S_{\exp }(t)-\left(\mathbf{k}(1) C_{I}(t)+\mathbf{k}(2) \int_{0}^{t} C_{I}(u) d u\right. \\
& \left.+(\mathbf{k}(2)-\mathbf{k}(3)) \int_{0}^{t} e^{-\mathbf{k}(4)(t-u)} C_{I}(u) d u\right),
\end{aligned}
$$

where $S_{\exp }$ is identified by the measured data, $C_{I}$ is the experimentally determined input function, and $\mathbf{k}=\left(w_{0}, \tilde{w}_{\mathrm{D}}, \tilde{w}_{\mathrm{A}}, \tilde{k}_{3}\right) \in \mathbb{R}_{+}^{4}$ is the vector of the unknowns. Therefore our aim is to determine $\mathbf{k}$ by the vanishing of $S_{t}$. This can be accomplished by means of the Newton method for multivariate functions, that reads:

$$
\left[\frac{d \mathcal{S}_{t}}{d \mathbf{k}}\left(\mathbf{k}^{(0)} ; \mathbf{h}^{(0)}\right)\right](t)=S_{\text {exp }}(t)-\mathcal{S}\left(\mathbf{k}^{(0)}\right)(t)
$$

with unknown $\mathbf{h}^{(0)} \in \mathbb{R}_{+}^{4}$, initial guess $\mathbf{k}^{(0)} \in \mathbb{R}_{+}^{4}$ and for all $t \in \mathbb{R}_{+}$. We therefore need to compute the Fréchet derivative at the left hand side of equation (13), which is possible since $\mathcal{S}_{t}$ can be easily seen to be differentiable and even analytic. For all $\mathbf{k} \in \mathbb{R}_{+}^{4}$, the Fréchet derivative $\frac{d \mathcal{F}}{d \mathbf{k}}(\mathbf{k})$ is the bounded and linear operator from $\mathbb{R}_{+}^{4}$ to $C^{1}\left(\mathbb{R}_{+}, \mathbb{R}\right)$ given by

$$
\begin{aligned}
\frac{d \mathcal{F}}{d \mathbf{k}}: \mathbb{R}^{4} & \rightarrow C^{1}\left(\mathbb{R}_{+}, \mathbb{R}\right), \\
\mathbf{h} & \mapsto\left(t \mapsto \nabla \mathcal{S}_{t}(\mathbf{k}) \cdot \mathbf{h}\right) .
\end{aligned}
$$

For all $t \in \mathbb{R}_{+}$, the gradient of $S_{t}$ is then given by

$$
\nabla \mathcal{S}_{t}(\mathbf{k})=\left(\begin{array}{c}
\frac{\partial \mathcal{S}_{t}}{\partial \mathbf{k}(1)} \\
\frac{\partial \mathcal{S}_{t}}{\partial \mathbf{k}(2)} \\
\frac{\partial \mathcal{S}_{t}}{\partial \mathbf{k}(3)} \\
\frac{\partial \mathcal{S}_{t}}{\partial \mathbf{k}(4)}
\end{array}\right)(\mathbf{k})=\left(\begin{array}{c}
-C_{I}(t) \\
-\left(\int_{0}^{t} C_{I}(u) d u+e^{-\mathbf{k}(4) t} \int_{0}^{t} e^{\mathbf{k}(4) u} C_{I}(u) d u\right) \\
e^{-\mathbf{k}(4) t} \int_{0}^{t} e^{\mathbf{k}(4) u} C_{I}(u) d u \\
(\mathbf{k}(2)-\mathbf{k}(3)) e^{-\mathbf{k}(4) t}\left(t \int_{0}^{t} e^{\mathbf{k}(4) u}\left(C_{I}(u)-u C_{I}(u)\right) d u\right)
\end{array}\right) .
$$

Equation (13) may have no solution and, furthermore, in real applications only noisy versions of $S_{\text {exp }}(t)$ for a finite number of sampling time points $t_{1}, \ldots, t_{n} \in \mathbb{R}_{+}$are available. It is therefore convenient to introduce a final regularization step to reduce the numerical instability which is intrinsic to these problems. To this aim we observe that the (non-regularized) discretized Multivariate Newton algorithm can be written as

$$
\left(\begin{array}{c}
{\left[\nabla \mathcal{S}_{t_{1}}\left(\mathbf{k}^{(0)}\right)\right]^{T}} \\
\vdots \\
{\left[\nabla \mathcal{S}_{t_{n}}\left(\mathbf{k}^{(0)}\right)\right]^{T}}
\end{array}\right) \mathbf{h}^{(0)}=\left(\begin{array}{c}
S_{\exp }\left(t_{1}\right)-\mathcal{S}_{t_{1}}\left(\mathbf{k}^{(0)}\right) \\
\vdots \\
S_{\exp }\left(t_{n}\right)-\mathcal{S}_{t_{n}}\left(\mathbf{k}^{(0)}\right)
\end{array}\right)
$$


i.e., in a compact form, as

$$
A^{(0)} \mathbf{h}^{(0)}=Y^{(0)}
$$

where $A_{0}$ is the matrix

$$
A^{(0)}=\left(\begin{array}{c}
{\left[\nabla \mathcal{S}_{t_{1}}\left(\mathbf{k}^{(0)}\right)\right]^{T}} \\
\vdots \\
{\left[\nabla \mathcal{S}_{t_{n}}\left(\mathbf{k}^{(0)}\right)\right]^{T}}
\end{array}\right)
$$

and $Y^{(0)}$ is the vector

$$
Y^{(0)}=\left(\begin{array}{c}
S_{\text {exp }}\left(t_{1}\right)-\mathcal{S}_{t_{1}}\left(\mathbf{k}^{(0)}\right) \\
\vdots \\
S_{\text {exp }}\left(t_{n}\right)-\mathcal{S}_{t_{n}}\left(\mathbf{k}^{(0)}\right)
\end{array}\right)
$$

Solving (15) by Tikhonov regularization consists in finding the solution $\mathbf{h}^{(0)}$ to

$$
\left(r I+\left(A^{(0)}\right)^{T} A^{(0)}\right) \mathbf{h}^{(0)}=\left(A^{(0)}\right)^{T} Y^{(0)},
$$

where $r$ is a regularization parameter. The value $\mathbf{k}^{(0)}$ is incremented, by letting $\mathbf{k}^{(1)}=\mathbf{k}^{(0)}+\mathbf{h}^{(0)}$ and the process is iterated [9]. Equation (18) defines the MRN algorithm.

\section{Numerical validation}

We describe the performance of our approach to compartmental analysis in the case of synthetic data simulated by mimicking a data acquisition process for Fmiso-based experiments using an instrument for small animals (the reference instrument is the micro-PET 'Albira' [11], Bruker Corporation, IRCCS San Martino IST, Genova).

\begin{tabular}{c|c|c|c|c}
\hline & $w_{0}$ & $\tilde{w}_{D}$ & $\tilde{w}_{A}$ & $\tilde{k}_{3}$ \\
\hline g. t. & 1.50 & 0.13 & 0.31 & 0.07 \\
\hline MRN & $1.49 \pm 0.02$ & $0.13 \pm 0.01$ & $0.31 \pm 0.02$ & $0.08 \pm 0.02$ \\
\hline LM & $1.47 \pm 0.10$ & $0.13 \pm 0.04$ & $0.30 \pm 0.06$ & $0.07 \pm 0.04$ \\
\hline \hline g. t. & 0.32 & 0.28 & 0.08 & 0.21 \\
\hline MRN & $0.31 \pm 0.02$ & $0.29 \pm 0.02$ & $0.08 \pm 0.00$ & $0.22 \pm 0.04$ \\
\hline LM & $0.33 \pm 0.06$ & $0.29 \pm 0.03$ & $0.08 \pm 0.01$ & $0.21 \pm 0.08$ \\
\hline \hline g. t. & 0.16 & 0.14 & 0.01 & 0.36 \\
\hline MRN & $0.16 \pm 0.01$ & $0.13 \pm 0.01$ & $0.01 \pm 0.00$ & $0.34 \pm 0.04$ \\
\hline LM & $0.16 \pm 0.02$ & $0.14 \pm 0.03$ & $0.01 \pm 0.00$ & $0.36 \pm 0.08$ \\
\hline
\end{tabular}

In order to simulate the synthetic data, we initially chose meaningful values for the four compartmental parameters $\left(w_{0}, \tilde{w}_{\mathrm{D}}, \tilde{w}_{\mathrm{A}}, \tilde{k}_{3}\right)$, and solve equation (9) to obtain estimate of $S(t)$, that is then sampled in time, on a time interval $\left[t_{1}, t_{n}\right]$ corresponding to the typical total acquisition time for Fmiso-PET experiments with 'Albira'. The IF $C_{I}$ has been obtained by fitting with a gamma variate function a set of real measurements acquired from a healthy mouse in a very controlled experiment [12]. Then we affected $S$ with Poisson noise, in order to have synthetic (but realistic) data. We finally applied our inversion algorithm for 50 runs in correspondence with 50 random values for the initial guess of the MRN scheme, for three different synthetic sets of values for the parameters (highly realistic, see [6]). The results of this test are given in Table 1, where the uncertainties on the reconstructed parameters are obtained by 
using the outcomes of the different runs. In the Table we report the results obtained by means of the LM technique for least-squares minimization. Comparison with respect to the ground-truth values and the values provided by the LM method and the small values for the reconstruction uncertainties shows the reliability of our approach. The results are also shown in Figure 2, where it is possible to observe how the MRN reconstructs time-concentration curves that are closer to the ground truth.
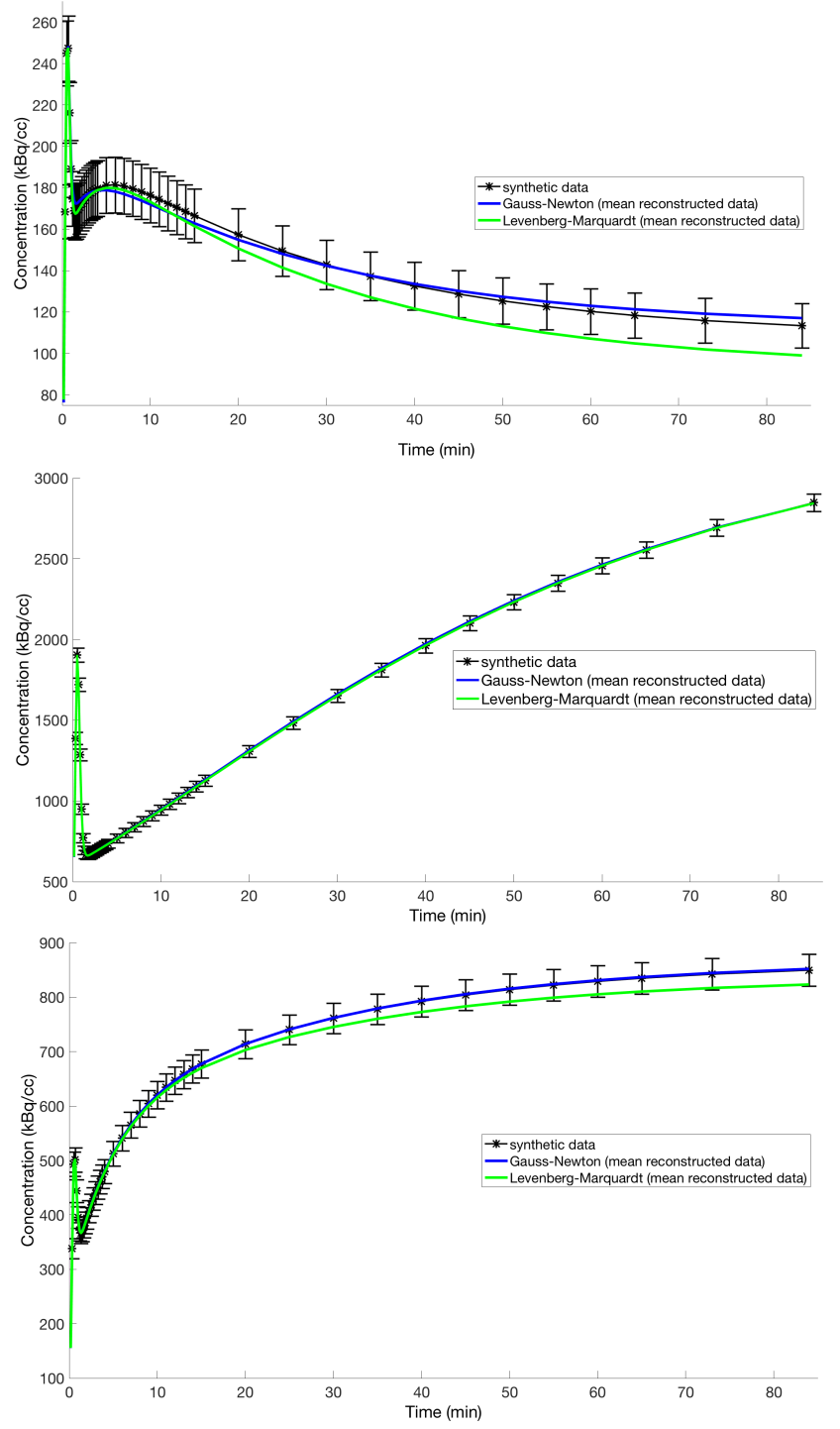

Figure 2. Simulated experiments with the parameters' values as in Table 1 . In each figure, the black line represents the synthetic signal (together with its confidence band); the blue line represents the time-concentration curve reconstructed by means of our Multivariate Regularized Newton method and the green line represents the one reconstructed via LevenbergMarquardt.

\section{Conclusion and discussion}

We described the performance of a regularized Newton approach to the numerical solution of the inverse problem associated to a compartmental model for Fmiso metabolism in PET experiments. The model assumes two compartments for the diffusive and the accumulative compartment, and is completely general, i.e. it is able to determine with a notable level of robustness and reliability all four compartmental parameters embedded in the model. A Matlab prototype implementing this approach is available at http: //mida.dima.unige.it/ together with a Graphical User Interface for user-friendly input/output processing. 


\section{References}

1. K. W-j, J. S. Rasey, M. L. Evans, J. R. Grierson, T. K. Lewellen, M. M. K. K. A. Graham, and G. T. $\mathrm{W}$, Imaging of hypoxia in human tumors with [f-18]fluoromisonidazole int, J. Radiat. Oncol. Biol. Phys., vol. 22, pp. 199-212, 1992.

2. J. S. Rasey, K. W-j, M. L. Evans, L. M. Peterson, T. K. G. M. M. Lewellen, and K. K. A, Quantifying regional hypoxia in human tumors with positron emission tomography of [18f]fluoromisonidazole: a pretherapystudy of 37 patients int, J. Radiat. Oncol. Biol. Phys., vol. 36, pp. 417-28, 1996.

3. M. Nordsmark, M. Overgaard, and J. Overgaard, Pretreatment oxygenation predicts radiation response in advanced squamous cell carcinoma of the head and neck radiother, Oncol., vol. 41, pp. 31-9, 1996.

4. M. Nordsmark and J. Overgaard, A confirmatory prognostic study on oxygenation status and locoregional control in advanced head and neck squamous cell carcinoma treated by radiation therapy radiother, Oncol., vol. 57, pp. 39-43, 2000.

5. J. J. G. M. M. Casciari and R. J. S, A modeling approach for quantifying tumor hypoxia with [f-18]fluoromisonidazole pet time-activity data, Phys. Med., vol. 22, pp. 1127-39, 1995.

6. D. Thorwarth, S. M. Eschmann, F. Paulsen, and M. Alber, A kinetic model for dynamic [18f]-fmiso pet data to analyse tumour hypoxia, Phys. Med., pp. 2209-24, 2005.

7. F. Delbary, S. Garbarino, and V. Vivaldi, Compartmental analysis of dynamic nuclear medicine data: models and identifiability, Inverse Problems, vol. 32, no. 12, p. 125010, 2016.

8. N. M. Alpert, R. D. Badgaiyan, and E. F. A. J. Livni, A novel method for noninvasive detection of neuromodulatory changes in specific neurotransmitter systems, Neuroimage, vol. 19, pp. 1049-60, 2003.

9. F. Delbary and S. Garbarino, Compartmental analysis of dynamic nuclear medicine data: regularization procedure and application to physiology arxiv, Inverse Problems in Science and Engineering, vol. 0, pp. 1-19, 2019.

10. H. M. Hudson and R. S. Larkin, Accelerated image reconstruction ordered subsets of projection data,, IEEE Trans Med Imaging, vol. 13, pp. 601-9, 1994.

11. T. Sasser, Preclinical imaging: improving translational power in oncology drug discovery, Drug Discovery, vol. 1, 2016.

12. S. R. Golish, J. D. Hove, and H. R. G. S. S. Schelbert, A fast nonlinear method for parametric imaging of myocardial perfusion by dynamic 13n- ammonia pet, J. Nucl. Med, vol. 42, pp. 924-31, 2001. 